\title{
BIOLOGÍA REPRODUCTIVA DE Sternula lorata, “GAVIOTÍN PERUANO”, EN LA RESERVA NACIONAL DE PARACAS (RNP), ICA - PERÚ
}

\section{BREEDING BIOLOGY OF Sternula lorata, PERUVIAN TERN, PARACAS NATIONAL RESERVE (PNR), ICA - PERU}

\author{
Samuel Amorós Kohn ${ }^{1}$, Patricia Saravia Guevara ${ }^{2}$ y Marta Williams ${ }^{3}$
}

\section{Resumen}

El "gaviotín peruano" es un ave de distribución restringida a la Corriente de Humboldt. Sus poblaciones a nivel mundial están disminuyendo, estimándose que existen de 1000 a 2499 individuos.

La investigación se desarrolló en la península de Paracas de noviembre 2006 a julio de 2008. Se ubicó, caracterizó los nidos y se describió la biología reproductiva de la especie. Se encontraron dos lugares de reproducción del "gaviotín peruano": 1) Pampa Lechuza y 2) Pampa Atenas Cequión. En ambos lugares, y como resultado acumulado de las dos temporadas reproductivas, se encontraron 60 nidos. Los nidos consisten en una depresión de $10.46 \mathrm{~cm}(\mathrm{n}=44, \pm 1.89)$ de diámetro mayor por $9.56 \mathrm{~cm}(\mathrm{n}=44, \pm 1.70)$ de diámetro menor y una profundidad de $1.56 \mathrm{~cm}$ $(\mathrm{n}=44, \pm 0.44)$. Estos fueron encontrados en lugares con una altitud menor a los $100 \mathrm{msnm} y$ estuvieron asociados a la presencia de piedras, huesos, conchales y/o alguna combinación de estos elementos. La distancia entre dos nidos activos varió desde $44.18 \mathrm{~m}$ hasta $3.28 \mathrm{~km}$ y la distancia de los nidos al mar varió entre $1 \mathrm{~km}$ a $3.7 \mathrm{~km}$. Esta especie pone principalmente de 1 a 2 huevos por nido. Los huevos presentaron $31.84 \mathrm{~mm}(\mathrm{n}=82 \pm 1.44)$ de largo por $23.88 \mathrm{~mm}(\mathrm{n}=82 \pm 0.82)$ de ancho. El éxito reproductivo de la temporada reproductiva 2006 - 2007 fue de $55.6 \%$ para nidos en los que por lo menos eclosionó un huevo. El "gaviotín peruano" tiene fidelidad al nido y/o al lugar de anidación y presenta una asincronía en su reproducción.

Palabras clave: Biología reproductiva, gaviotín peruano, Sternula lorata, Paracas, éxito reproductivo y características de los nidos.

\begin{abstract}
The Peruvian Tern is an endemic bird to the Humboldt Current. Its population, currently estimated to be from 1000 to 2499 individuals is declining worldwide.

The research was conducted at the Paracas Peninsula from November 2006 to July 2008. Nests were located and marked and the reproductive biology of the species was described. Two breeding sites of the Peruvian Tern were located: 1) Pampa Lechuza and 2) Pampa Atenas Cequión. In both places, and as a cumulative result of the two breeding seasons, 60 nests were found. The nests consist of a depression with the following dimensions: $10.46 \mathrm{~cm}(\mathrm{n}=44 \pm 1.89)$ of greater diameter; $9.56 \mathrm{~cm}(\mathrm{n}=44 \pm 1.70)$ of smaller diameter and $1.56 \mathrm{~cm}(\mathrm{n}=44 \pm 0.44)$ of depth. They were found in areas with an altitude less than 100 m.a.s.l. and were associated with the presence of stones, bones, shell and / or some combination of these elements. The distance between two active nests varied from $44.18 \mathrm{~m}$ to $3.28 \mathrm{~km}$ and the distance of nests to the sea varied from $1 \mathrm{~km}$ to $3.7 \mathrm{~km}$. This species lays mostly 1 to 2 eggs per nest. The eggs were 31.84 $\mathrm{mm}(\mathrm{n}=82 \pm 1.44)$ long by $23.88 \mathrm{~mm}(\mathrm{n}=82 \pm 0.82)$ wide. The reproductive success of the $2006-$ 2007 breeding season was 55.6 \% for those nests in which at least one egg hatched. The Peruvian Tern has fidelity to the nest and / or nesting site and presents an asynchrony in reproduction.

Key words: Reproductive biology, Peruvian tern, Sternula lorata, Paracas, reproductive success and characteristics of the nests.
\end{abstract}

\section{Introducción.}

El "gaviotín peruano" (Sternula lorata) es una especie endémica de la corriente de Humboldt (Vilina, 1998). Su distribución norte llega hasta La Isla El Muerto en el Golfo de Guayaquil, Ecuador, y por el sur llega hasta Antofagasta en Chile (Murphy, 1936; Harrison, 1985; Vilina, 1998). Sus lugares de reproducción se caracterizan por ser espacios abiertos, no protegidos, en áreas planas del desierto, usualmente ubicados entre 1 y $2 \mathrm{~km}$ tierra adentro (Murphy, 1936; Johnson, 1967; Vilina, 1998). Los sustratos son generalmente arenosos o grava fina, con distintas densidades de rocas pequeñas a medianas, dispersas por el lugar (Guerra-Correa, 2003). En Perú se ha encontrado que estos lugares pueden presentar 
variaciones entre localidades siendo algunos de ellos playas de arena asociadas a humedales (Zavalaga et al., 2008b). Esta ave se diferencia de otras aves marinas porque es la única que utiliza el desierto costero o tablazo para reproducirse (Zavalaga et al., 2005; Tello et al., 2008). El “gaviotín peruano” es una especie que ubica sus nidos en el suelo, sin adornos, $\mathrm{y}$ pone de 1 a 2 huevos muy crípticos (Guerra-Correa, 2005). Los nidos tienen un diámetro de $109 \pm 16.93$ $\mathrm{mm}(\mathrm{n}=9)$ y una profundidad de $15.8 \pm 2.28 \mathrm{~mm}(\mathrm{n}=$ 5) y pueden ser encontrados junto a huesos, restos de bivalvos o piedras (del Hoyo et al., 1996; Zavalaga et al., 2008a). Después de un periodo de incubación de 22 a 23 días, el pichón eclosiona del huevo con un peso de alrededor de $7 \mathrm{~g}$ con un color gris pálido y finamente moteado en la cabeza y dorso (del Hoyo et al., 1996). El "gaviotín peruano" presenta una asincronía en la fecha de puesta de los huevos (Vilina, 1998; Zavalaga et al., 2005). Se reproduce de octubre a enero y el pico reproductivo según del Hoyo et al. (1996) se presenta en noviembre.

El "gaviotín peruano" es una especie amenazada en todo su ámbito de distribución y está categorizada internacionalmente como especie "En Peligro" (Endangered, EN; BirdLife Internacional, 2009) у а nivel nacional como especie vulnerable (Decreto Supremo $\mathrm{N}^{\circ}$ 034-2004-AG). Su población total fue estimada en 10000 individuos (Duffy et al., 1984) y actualmente se estima que a nivel mundial su población varía entre 1000 a 2499 individuos (BirdLife Internacional, 2009). Actualmente se conocen 4 sitios de nidificación en el Perú, los cuales son Pacasmayo, Paraíso, Pampa Lechuza y Tres Hermanas-Yanyarina; y 8 en Chile, ubicados en la península de Mejillones y áreas cercanas (GuerraCorrea, 2003; Zavalaga et al., 2009).

La península de Paracas se encuentra en la Reserva Nacional de Paracas, la cual es un Área Natural Protegida por el Estado que conserva una muestra representativa de los ecosistemas marino-costeros del Perú (INRENA, 2002). Esta Reserva reúne diversos valores históricos, biológicos, culturales y económicos que hacen de ella un lugar único en todo el litoral peruano (Reyes \& Hammann, 2002). En el presente trabajo se caracterizan los lugares de reproducción del “gaviotín peruano" en la península de Paracas y se ubica y evalúa el tamaño de los mismos. La generación de mayor conocimiento sobre la biología reproductiva de esta especie contribuirá al desarrollo de acciones que garanticen la conservación del "gaviotín peruano", especie amenazada que constituye un objeto de conservación para la RNP (INRENA, 2002).

\section{Materiales y métodos.}

\section{Área de estudio.}

La península de Paracas se ubica en la zona norte de la Reserva Nacional de Paracas. La RNP se encuentra entre los paralelos $13^{\circ} 47^{\prime}$ S y $14^{\circ} 26^{\prime}$ S y los 76 $30^{\circ}$ 'W y los $76^{\circ} 00^{\prime} \mathrm{W}$. Políticamente, la Reserva se ubica en el departamento de Ica, provincias de Pisco e Ica, distritos de Paracas y Salas, respectivamente (INRENA, 2002).

El trabajo de campo se realizó desde noviembre 2006 hasta julio 2008. Las observaciones para la búsqueda de nidos se realizaron durante los periodos de luz y en grupos de 2 a 4 personas. El esfuerzo de muestreo fue más intenso entre noviembre 2006 y mayo 2007, periodo que correspondió a la temporada reproductiva 2006-2007 y en donde las salidas de campo se realizaron cada 15 días aproximadamente; luego de este periodo, las salidas de campo fueron mensuales o bimestrales. Para el cálculo de la distancia, entre nidos y de los nidos respecto al mar, se utilizó la distancia euclidiana y el programa ArcView GIS 3.2. Las medidas son presentadas con su respectiva desviación estándar ( \pm D.S.).

Se revisaron los lugares potenciales de reproducción del "gaviotín peruano" mediante recorridos a pie por el desierto en grupos de 2 a 4 personas en los cuales cada observador se desplazó por un transecto. La separación entre transectos fue de máximo $100 \mathrm{~m}$ y se distribuyeron en forma paralela. Cada nido fue georreferenciado con un navegador GPS, se midió el diámetro mayor, diámetro menor y la profundidad de estos con una regla y/o cinta métrica (wincha) $( \pm 1 \mathrm{~mm})$. También se describió el nido tomando en cuenta el tipo y color de sustrato en el que se encontró, presencia de piedras, altitud y las distancias anteriormente mencionadas.

Sólo se consideró como nidos de "gaviotín peruano" a aquellos que tuvieron presencia de huevos y/o pichones en su interior. En un sólo caso se consideró como un nido una depresión con características similares a la de los otros nidos y en cuyo interior se encontró restos de alimentos y heces. Los nidos fueron caracterizados en dos clases según el grado de actividad que presentaron al momento de ser encontrados. Estas clases fueron: 1) Nidos activos: aquellos que presentaron huevos puestos en la temporada de evaluación y/o presentaron pichones; y 2) Nidos inactivos: aquellos que presentaron huevos de temporadas anteriores o que en el momento de su hallazgo los huevos ya habían sido abandonados. También, en cada nido se describió las características de los huevos y/o pichones encontrados y se tomaron las medidas biométricas de cada uno de estos. Para el caso de los huevos, estas medidas consistieron en: peso con balanza de resorte (pesola) de $30 \mathrm{~g}( \pm 0.25)$, y largo y ancho con calibrador (vernier) ( $\pm 0.1 \mathrm{~mm})$; mientras que para los pichones, consistieron en: peso con balanza de resorte de $30 \mathrm{~g}( \pm 0.25)$ y $60 \mathrm{~g}( \pm 0.50)$, longitud del culmen (pico completo) y longitud del tarso con calibrador ( $\pm 0.1 \mathrm{~mm})$, y longitud del ala con una regla con tope $( \pm 1 \mathrm{~mm})$. 


\begin{abstract}
El éxito
reproductivo calculado considerando la proporción de nidos en los que al menos eclosionó un huevo y también tomando en cuenta la cantidad de huevos eclosionados respecto al número de huevos totales puestos en el nido. Durante cada salida de campo se buscaron nuevos nidos y se revisaron los nidos hallados en las salidas anteriores.
\end{abstract}

\section{Resultados discusión.}

Se realizaron un total de 36 salidas de campo entre noviembre

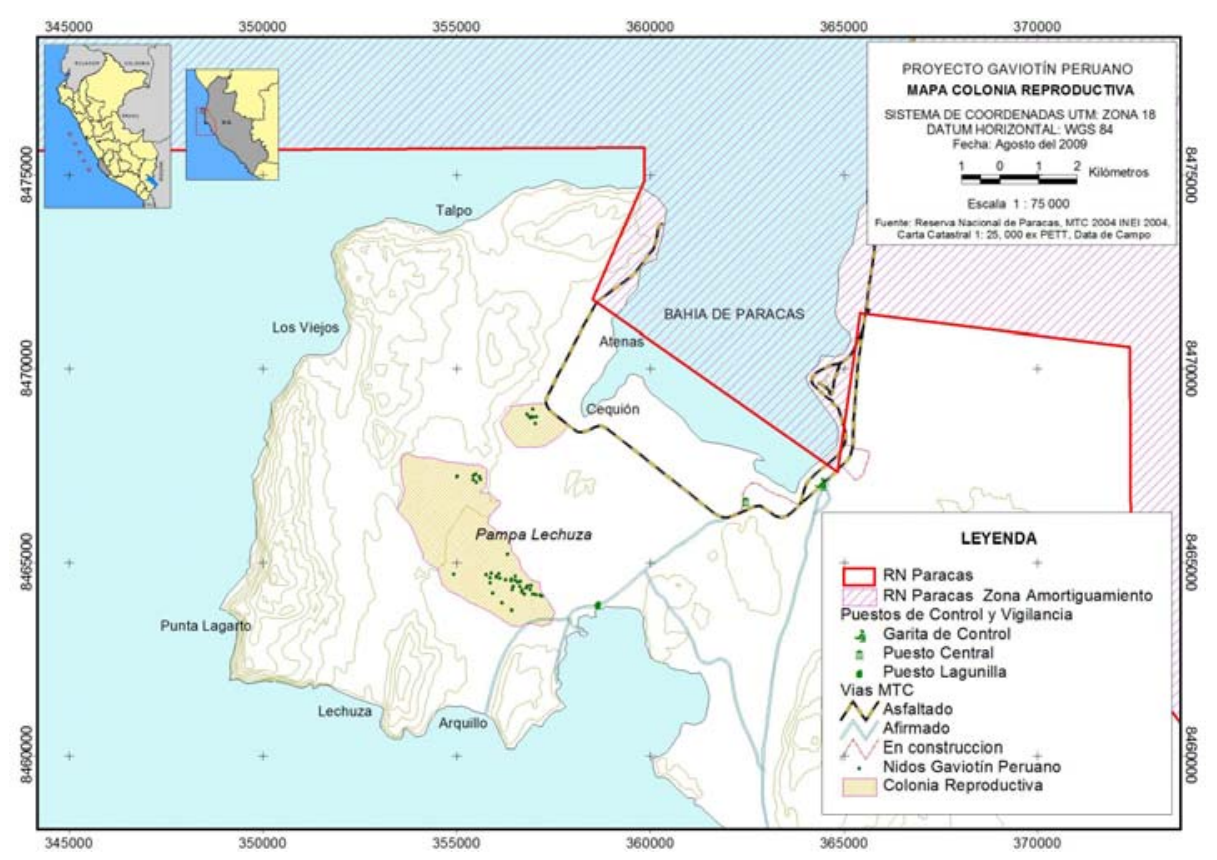

Figura 1. Colonia reproductiva de Sternula lorata, "gaviotín peruano"

2006 a julio 2008; lo que representa un aproximado de 167 días/hombre, de los cuales 118 correspondieron a la temporada reproductiva 2006-2007 (noviembre 2006 - mayo 2007). Durante los dos primeros meses se buscó nidos en los lugares potenciales de reproducción de esta especie, estos fueron: Pampa Arquillo, Pampa Lechuza, Pampa Atenas, Cequión, Punta Ballenas, Cangrejal y La Aguada. Luego se determinó, en base a los hallazgos, que el "gaviotín peruano” tiene preferencia por las zonas comprendidas entre Pampa Arquillo y Pampa Lechuza. Asimismo, en el 2008 se observó que el "gaviotín peruano" también utilizó como área de reproducción la zona ubicada cerca de pampa Atenas - Cequión (zona centro Oeste de la península de Paracas, a la cual se le denominará Pampa Atenas - Cequión).

Colonia reproductiva.

La colonia reproductiva del "gaviotín peruano” fue encontrada en Pampa Lechuza y en Pampa Atenas Cequión, ambas ubicadas al interior de la península de Paracas, dentro de la Reserva Nacional de Paracas (Figura 1). Estos lugares se caracterizan por ser zonas de escasa pendiente con presencia de piedras y suelo mayormente arenoso (arena gruesa sobre una costra salina “caliche”). También se observó que al interior de estas áreas reproductivas se encuentra una serie de vías o caminos (trochas no oficiales), las cuales son utilizadas por diferentes actores (pescadores, personal de la Reserva, turistas, investigadores, entre otros) para desplazarse a distintos lugares de la Península. Para el cálculo de la extensión de estas áreas de reproducción se ha considerado que éstas incluyan los lugares con características similares a las zonas donde se encontraron los nidos del "gaviotín peruano" y/o lugares de descanso de la especie. En el caso de la zona reproductiva de Pampa Lechuza sus límites han sido ampliados hasta que colinden con alguna vía (trocha) de tal manera que pueda, en un futuro, desarrollarse un plan de señalización y se regule el tránsito vehicular por el interior de la colonia reproductiva de este gaviotín. En el caso de la zona reproductiva de Pampa Atenas - Cequión, los límites de ésta también incluyen los lugares con características similares a la de los nidos y, a diferencia de la zona reproductiva de Pampa Lechuza, no presenta vías cercanas en su periferia que puedan servir como límites referenciales para el cálculo de su extensión; por lo tanto, sus limites han sido ampliados entre 150 a $200 \mathrm{~m}$ desde el punto más distante que presenta características similares a la zona de los nidos de la especie de estudio. De esta manera, aproximadamente la extensión de la zona reproductiva de Pampa Lechuza es de 950 ha y la extensión de la zona reproductiva de Pampa Atenas - Cequión es de 140 ha.

Características de los nidos-

Los nidos consisten en una pequeña depresión de unos $10.46 \mathrm{~cm}(\mathrm{n}=44, \pm 1.89)$ de diámetro mayor por unos $9.56 \mathrm{~cm}(\mathrm{n}=44, \pm 1.70)$ de diámetro menor y una profundidad de $1.56 \mathrm{~cm}(\mathrm{n}=44, \pm 0.44)$. Estos nidos no presentan mayor decoración como puede verse en la Figura 2. Todos los nidos se encontraron en zonas planas con presencia de piedras de formas irregulares y de tamaños de hasta $20 \mathrm{~cm}$ aproximadamente. El tipo de sustrato mayormente fue arenoso con presencia de piedritas (gravillas y arenas gruesas) sobre una costra salina; sólo 2 nidos se encontraron en una zona con restos de conchales.

Considerando que los nidos se encontraron con presencia de piedras, huesos, conchales y/o con alguna 
combinación de estos elementos; se diseñó una metodología para su clasificación. Esta incluyó la descripción de los elementos más cercanos al nido hasta una distancia no mayor de 0.5 metros aproximadamente. También, para fines de la presente clasificación el término piedra en las categorías se refiere a todas aquellas cuyo tamaño sea mayor o igual a los $6 \mathrm{~cm}$. Esta clasificación consistió en:

a) Nido rodeado de piedras

Incluye a todos los nidos que presentan piedras alrededor o se encuentran parcialmente rodeados por éstas (Figura 2 a).

b) Nido junto a piedras

Son nidos donde las piedras se encuentran ubicadas a un lado de éste. Éstas pueden estar aisladas o agrupadas; sin embargo, en ambos casos no se encuentran alrededor del nido.

c) Nido sobre conchales

Son nidos que se encuentran en zonas donde predomina la presencia de restos de bivalvos y caracoles (Figura 2 b).

d) Nido junto a huesos

Son nidos que se encuentran junto a huesos, principalmente de aves. Estos nidos no presentan piedras (Figura $2 \mathrm{c}$ ).

e) Nido rodeado de piedras y huesos

Son nidos que presentan piedras $\mathrm{y}$ huesos alrededor o parcialmente alrededor de estos.

f) Nido junto a piedras y huesos

Son nidos donde las piedras y los huesos se encuentran a un lado del nido. Estos pueden estar aislados o agrupados; sin embargo, en ambos casos no están alrededor del nido.

Como se observa en la Tabla 1 , el $60 \%$ de los nidos estuvo rodeado de piedras y el $25 \%$ junto a piedras. En términos generales, cerca del 92\% estuvo asociado a la presencia de piedras, resaltándose la importancia de este elemento en la reproducción del "gaviotín peruano" ya que contribuye con la protección y camuflaje de los pichones.

Tabla 1. Frecuencia de nidos de Sternula lorata, "gaviotín peruano”, según las categorías encontradas en la península de Paracas (RNP, Ica Perú). Periodo de estudio: noviembre 2006 - julio 2008.

\begin{tabular}{lcc}
\hline Categoría de Nidos & $\begin{array}{c}\text { Frecuencia } \\
\left(\mathrm{N}^{\mathrm{o}} \text { Nidos }\right)\end{array}$ & Porcentaje \\
\hline Rodeado de piedras & 36 & 60.0 \\
Junto a piedras & 15 & 25.0 \\
Sobre de conchales & 2 & 3.3 \\
Junto a huesos & 3 & 5.0 \\
Rodeado de piedras y huesos & 1 & 1.7 \\
Junto a piedras y huesos & 3 & 5.0 \\
\multicolumn{1}{c}{ TOTAL } & 60 & \\
\hline
\end{tabular}

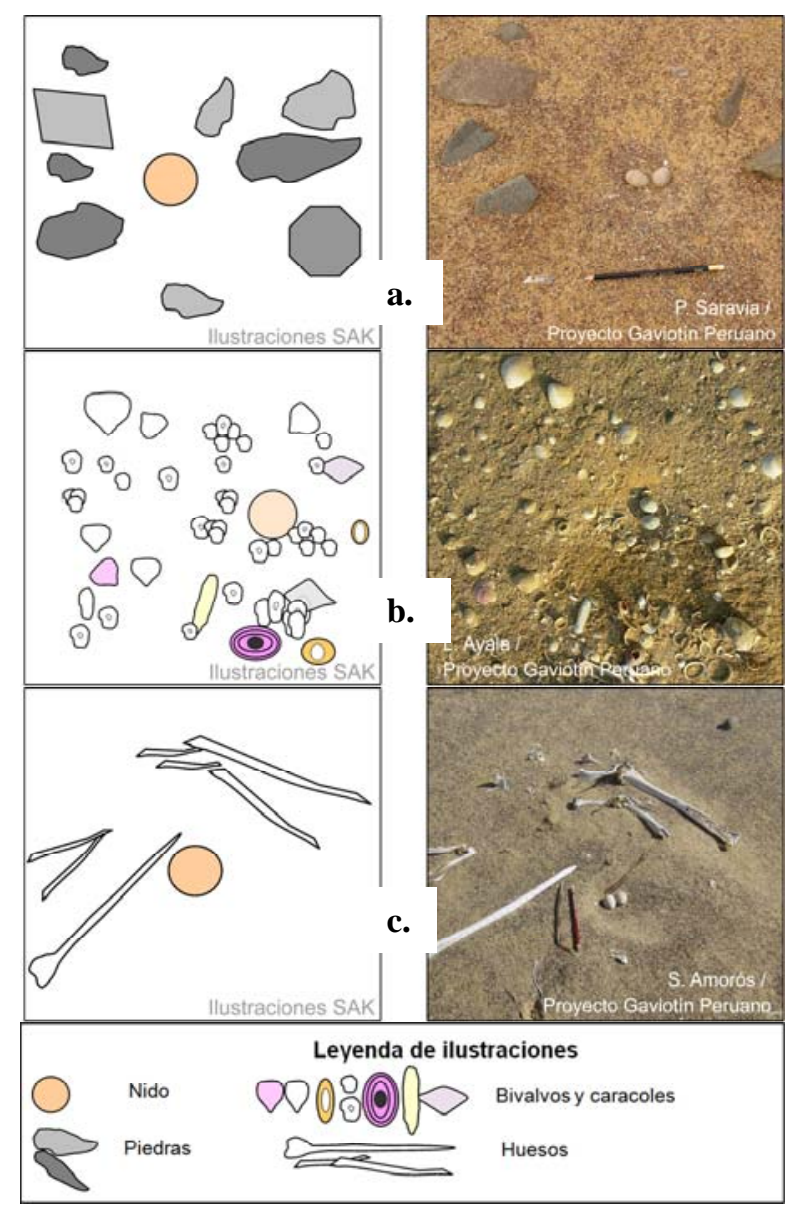

Figura 2. Nidos de Sternula lorata "gaviotín peruano”
a. Nido rodeado de piedras.
b. Nido sobre conchales.
c. Nido junto a huesos.

Todos los nidos se encontraron en zonas planas con una altitud menor a los $100 \mathrm{msnm}$. La altitud de cada uno de los nidos fue medida con un navegador GPS cuyo margen de error pudo variar entre $\pm 15 \mathrm{~m}$. La altitud promedio de los nidos fue de 43.6 m.s.n.m. ( $\mathrm{n}=59$, $\pm 19.5 \mathrm{msnm}$ ), con un valor mínimo de 12.1 m.s.n.m. y un valor máximo de 85 msnm.

El color del suelo de los nidos fue estudiado en base al Sistema Munsell y los resultados pueden observarse en la Tabla 2.

El número total de nidos encontrados desde noviembre del 2006 a julio del 2008 fue de 60 nidos, la proporción de nidos activos y nidos inactivos encontrados puede observarse en la Tabla 3. El mayor número de nidos, tanto para la temporada 2006-2007 como para la 2007-2008, se encontró en el mes de enero 
Tabla 2. Clases de color del sustrato de los nidos de Sternula lorata "gaviotín peruano" (n= 37) en la península de Paracas. Temporada reproductiva: 2006 - 2007 (RNP, Ica - Perú).

\begin{tabular}{lc}
\hline Color & Frecuencia \\
\hline Amarillo & 2 \\
Amarillo olivo & 12 \\
Amarillo pálido & 4 \\
Pardo ligeramente amarillento & 14 \\
Pardo ligeramente olivo & 5 \\
\hline
\end{tabular}

La separación más pequeña entre 2 nidos activos (nidos con presencia de huevos cuya fecha de puesta fue hasta un máximo de 4 semanas o nidos con pichones aún en el nido) fue de $44.18 \mathrm{~m}$, mientras que la mayor distancia entre este tipo de nidos fue de 3.28 km. La distancia de los nidos al mar varió aproximadamente desde $1 \mathrm{~km}$ hasta los $3.7 \mathrm{~km}$ para el caso de la zona reproductiva de Pampa Lechuza y de $1.2 \mathrm{~km}$ a $1.4 \mathrm{~km}$ para el caso de la zona reproductiva de Pampa Atenas - Cequión.

Descripción de la biología reproductiva.

Durante la temporada 2006-2007, los nidos hallados presentaron nidadas que variaron principalmente entre 1 a 2 huevos, los cuales fueron puestos en diferentes tiempos; en un sólo caso se encontró un nido con 3 huevos. En la temporada 20072008, se encontró también un nido con 3 huevos; sin embargo, uno de ellos correspondía a la temporada reproductiva anterior (2006-2007). En ambas temporadas reproductivas se encontró que el 59.6\% de las nidadas fueron de 2 huevos, el 36.2\% fueron de un sólo huevo y tan sólo el $2.1 \%$ fue de 3 huevos.

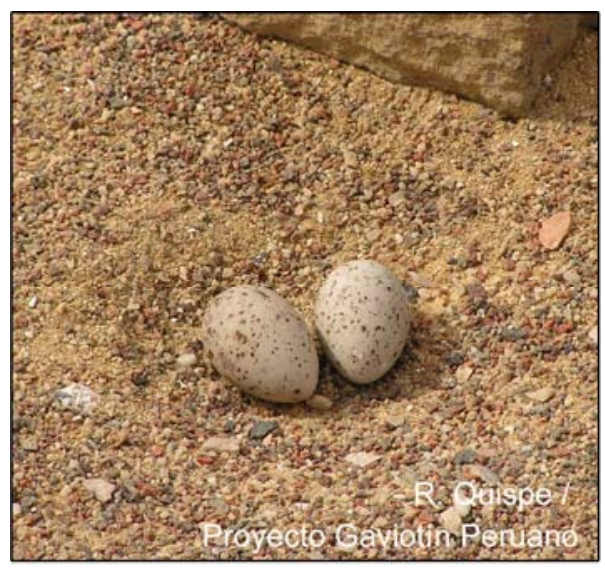

Figura 3. Mimetización de huevos de Sternula lorata "gaviotín peruano" en el desierto.

Los huevos son de color crema con presencia de puntos de color marrón oscuro lo cual permite que se mimeticen en el desierto como se muestran en la Figura 3. Los huevos se caracterizaron por presentar 2 tipos de forma: huevos ligeramente esféricos y huevos ligeramente en punta. El color de estos varió
Tabla 3. Nidos activos e inactivos de Sternula lorata, "gaviotín peruano", por temporada reproductiva en la península de Paracas (RNP, Ica - Perú).

\begin{tabular}{cccc}
\hline Temporada & $\begin{array}{c}\text { Nidos } \\
\text { Activos }\end{array}$ & $\begin{array}{c}\text { Nidos } \\
\text { Inactivos }\end{array}$ & Total \\
\hline $2006-2007$ & 29 & 8 & 37 \\
$2007-2008^{*}$ & 12 & $11^{* *}$ & 23 \\
\hline
\end{tabular}

(*) Observaciones incompletas de temporada reproductiva 2007-2008.

(**) Observaciones fuera del periodo reproductivo.

sutilmente según el tiempo en que fueron puestos. Los huevos recientes presentaron un color ligeramente verdoso, mientras que los huevos con más tiempo de puesta fueron ligeramente más crema. Las dimensiones de los huevos pueden observarse en la Tabla 4. El peso de los huevos varió según el tiempo, encontrándose que los huevos recién puestos (huevos verdosos) presentaron un peso de aproximadamente 9.29 g $( \pm 0.46, n=12)$ mientras que los huevos cerca a la eclosión (huevos rajados por el pichón) tuvieron un peso de $7.8 \mathrm{~g}( \pm 0.75, \mathrm{n}=6)$.

Tabla 4. Dimensión de los huevos de Sternula lorata, "gaviotín peruano". (n=82 huevos).

\begin{tabular}{lcc}
\hline & Largo $(\mathrm{mm})$ & Ancho $(\mathrm{mm})$ \\
\hline Promedio & 31.84 & 23.88 \\
Desviación estándar & 1.4487 & 0.8235 \\
Máximo & 34.5 & 25.5 \\
Mínimo & 28.1 & 22.5 \\
\hline
\end{tabular}

La característica precocial de los pichones de abandonar el nido a los pocos días de eclosionar, la asincronía en la reproducción y el intervalo entre cada salida de campo no permitió determinar la fecha de puesta de los huevos en el nido y su respectiva fecha de eclosión. Los pichones con una edad de máximo un día de eclosión presentaron el cuerpo cubierto de plumón con una ligera ausencia de éste en una parte del dorso. Las alas, cabeza y dorso tienen plumón de color beige con puntos negros, el pecho y vientre con plumón blanco. Las patas son de color rosado, la mandíbula inferior es ligeramente rosada, la mandíbula superior desde el inicio hasta las narinas es de color oscuro, la parte central de color rosado y la punta de la mandíbula inferior y superior son de color oscuro. Presentan diente de huevo. Las medidas biométricas de estos pichones se presentan en la Tabla 5.

El éxito reproductivo fue evaluado sólo en la temporada reproductiva 2006-2007. La proporción de nidos que lograron la eclosión de al menos un huevo fue de $55.56 \%$ ( $n=27$ nidos) mientras que el $14.81 \%$ fueron nidos donde los huevos no eclosionaron y en el 
$29.63 \%$ no pudo determinarse el éxito reproductivo por no encontrarse los huevos y/o pichones de estos nidos. También se calculó el éxito reproductivo considerando la cantidad de huevos eclosionados respecto al número de huevos puestos en el nido. En ese sentido, de un total de 27 nidos con huevos evaluados en la temporada 2006-2007 se tiene la certeza que en 11 de ellos se logró un éxito de eclosión del $100 \%$, en 4 de ellos el éxito fue del 50\%, es decir, sólo eclosionó un huevo de los 2 huevos colocados en el nido, en 4 de ellos el éxito de eclosión fue de $0 \%$ y en 8 nidos no pudo determinarse el éxito de eclosión debido a que no se encontraron los huevos y/o pichones de estos nidos.

Tabla 5. Dimensión de los pichones de Sternula lorata, "gaviotín peruano" con una edad de máximo un día ( $n=5$ pichones).

\begin{tabular}{lcccc}
\hline & $\begin{array}{c}\text { Peso } \\
(\mathrm{g})\end{array}$ & $\begin{array}{c}\text { Ala } \\
(\mathrm{cm})\end{array}$ & $\begin{array}{c}\text { Pico } \\
(\mathrm{cm})\end{array}$ & $\begin{array}{c}\text { Tarso } \\
(\mathrm{cm})\end{array}$ \\
\hline Promedio & 6.70 & 1.30 & 0.79 & 1.05 \\
Máximo & 7.3 & 1.4 & 0.9 & 1.3 \\
Mínimo & 6.0 & 1.1 & 0.7 & 1.0 \\
Desviación estándar & 0.54 & 0.14 & 0.08 & 0.12 \\
\hline
\end{tabular}

Durante el desarrollo de esta investigación se encontraron sólo 4 pichones muertos, en la temporada 2006-2007, 2 de los cuales pertenecían a 2 nidos monitoreados y los otros 2 no pudo determinarse a que nido correspondían.

Se encontró que el "gaviotín peruano" tiene fidelidad al nido y/o al lugar de anidación, es decir reutiliza sus mismos nidos, anida a escasos metros de sus nidos anteriores y/o anida en los mismos lugares que utilizó anteriormente. En la temporada 2006-2007 se encontraron 3 nidos reutilizados, es decir, nidos donde después de la eclosión o pérdida de los huevos fueron utilizados para nuevas nidadas. En la temporada 2007-2008, estudiada parcialmente, se encontraron 7 nidos reutilizados, los cuales habían sido usados en la temporada reproductiva anterior.

Se observó asincronía en la reproducción, es decir en un mismo periodo de tiempo se observó nidos con presencia de huevos y pichones como también presencia de volantones y juveniles. En la Tabla 6 se muestra la cronología de la reproducción del "gaviotín peruano” en la península de Paracas en la temporada reproductiva 2006-2007.

Tabla 6. Cronología de la reproducción de Sternula lorata, “gaviotín peruano”, en la península de Paracas (periodos en rangos de 15 días aproximadamente). Temporada reproductiva 2006-2007.

\begin{tabular}{lccccccccccc}
\hline & Diciembre & \multicolumn{2}{c}{ Enero } & \multicolumn{2}{c}{ Febrero } & \multicolumn{2}{c}{ Marzo } & \multicolumn{2}{c}{ Abril } & \multicolumn{2}{c}{ Mayo } \\
\hline Presencia de huevos & & $\mathrm{X}$ & $\mathrm{X}$ & $\mathrm{X}$ & $\mathrm{X}$ & $\mathrm{X}$ & $\mathrm{X}$ & $\mathrm{X}$ & $\mathrm{X}$ & $\mathrm{X}$ & \\
Presencia de pichones & & $\mathrm{X}$ & $\mathrm{X}$ & $\mathrm{X}$ & $\mathrm{X}$ & $\mathrm{X}$ & $\mathrm{X}$ & $\mathrm{X}$ & $\mathrm{X}$ & $\mathrm{X}$ \\
Presencia de volantones y juveniles & & $\mathrm{X}$ & $\mathrm{X}$ & $\mathrm{X}$ & $\mathrm{X}$ & $\mathrm{X}$ & $\mathrm{X}$ & $\mathrm{X}$ & $\mathrm{X}$ & $\mathrm{X}$ & $\mathrm{X}$ \\
\hline
\end{tabular}

\section{Discusión}

La zona de reproducción del "gaviotín peruano" fue encontrada en el desierto de la península de Paracas, en un lugar donde probablemente coincida con la observada por Murphy en 1920, quien la menciona como "la pampa”, y en la cual observó indicios de reproducción de esta ave. Sin embargo, pese a su búsqueda no logró encontrar nidos (Murphy, 1936). Los hallazgos de la presente investigación coinciden y complementan los reportados por otros investigadores quienes accidentalmente encontraron nidos de esta especie (A. Catenazzi com pers., guardaparques de la RNP com pers.), así como con las recientes publicaciones de Zavalaga et al. (2008a, 2008b y 2009).

Dadas las características de los nidos y sobre todo porque en la zona reproductiva, al interior de la península de Paracas, se observó que es común encontrar estructuras similares a los nidos de Sternula lorata sin llegar a serlo y que en algunos casos sólo constituyen lugares de descanso o dormideros de la especie, se prefirió sólo considerar como nido aquellos que presentaron huevos y/o pichones en su interior y sólo en un caso se consideró como nido (inactivo) al hallazgo de alimento seco y heces en una depresión de iguales características y dimensiones a la de los nidos encontrados. Asimismo, para el recuento de los nidos tampoco se ha considerado como tales a los pichones hallados deambulando en el desierto y cuyo nido de procedencia no pudo determinarse. Los nidos encontrados durante todo el periodo de estudio tuvieron presencia de piedras, huesos, conchales y/o alguna combinación de estos elementos. Asimismo, aproximadamente el $92 \%$ de los nidos estuvo asociado a las piedras resaltando así la importancia de este elemento en la reproducción, como también lo menciona Guerra-Correa (2003) en su estudio en la península de Mejillones en Chile. Aproximadamente el $40 \%$ de los nidos tuvo un sustrato de color pardo ligeramente amarillento y el $32 \%$ amarillo olivo. Estos colores del sustrato y la presencia de piedras permite que los huevos y pichones se mimeticen con el desierto dificultando el hallazgo y observación de los mismos. El periodo de incubación se calcula que pueda estar alrededor de las 3 semanas. Este valor no pudo determinarse con mayor exactitud por la característica precocial de los pichones de abandonar el nido a los pocos días de eclosión, la asincronía reproductiva y las frecuencias de las salidas de campo. Este cálculo es similar al mencionado por del Hoyo et 
al. (1996) quienes señalan que el periodo de incubación es de 22 a 23 días y también a lo mencionado por Zavalaga et al. (2008a), quienes señalan que en Puerto Viejo la moda del periodo de incubación calculada desde la fecha de encuentro del huevo hasta la eclosión fue de 22 días (rango: 22-23 días, $n=4)$.

Adicionalmente a los lugares de reproducción al interior del desierto costeño, se puede mencionar como otros hábitats reproductivos a los humedales marino costeros ya que existen reportes de zonas de reproducción de esta especie asociados a estos humedales, tales como en Paraíso (Tello et al., 2008), así como también en los de Puerto Viejo y Mollendo. Debido a la perturbación humana estas dos últimas zonas ya no son utilizadas por esta especie para reproducirse desde 1977 para el caso de Puerto Viejo y desde 1978 para Mollendo (Zavalaga et al., 2008b).

La colonia reproductiva encontrada en la península de Paracas, en Pampa Lechuza y Pampa Atenas Cequión, constituye un lugar de importancia para la reproducción del “gaviotín peruano”. Esta zona es permanentemente utilizada por esta especie como sitio reproductivo como lo puede corroborar la fidelidad al nido y/o al lugar de anidación que se encontró en la presente investigación así como las observaciones de Murphy en 1920 sobre indicios de reproducción en una zona que el denomina como "la pampa" (Murphy, 1936) y las observaciones de Coker en 1907 de abundantes individuos durante varios periodos del año en la Bahía de Paracas (Coker, 1919). Todo esto permitiría proponer que esta especie ha venido reproduciéndose en la península de Paracas desde por lo menos inicios de los 1900 hasta la actualidad. En ese sentido, es recomendable reforzar los esfuerzos de conservación en esta área natural protegida para garantizar la conservación del "gaviotín peruano" especie que se encuentra amenazada a nivel global y nacional por las perturbaciones en sus lugares reproductivos.

\section{Conclusiones.}

La colonia reproductiva de Sternula lorata fue encontrada en Pampa Lechuza y Pampa Atenas Cequión, ambos lugares en la península de Paracas al interior de la Reserva Nacional de Paracas. En la temporada reproductiva 2006-2007 se encontró 37 nidos y en la temporada reproductiva estudiada en forma parcial se encontraron 23. Los nidos se ubicaron en zonas planas con una altitud menor a los 100 msnm. Se caracterizaron por ser pequeñas depresiones de aproximadamente $10.46 \mathrm{~cm} \pm 1.89$ (rango: $9.5-$ $10, \mathrm{n}=44$ ) por $9.56 \mathrm{~cm} \pm 1.70$ (rango: $9.5-10, \mathrm{n}=44$ ) y $1.56 \mathrm{~cm} \pm 0.44$ (rango: $1.0-2.5, \mathrm{n}=44$ ) de profundidad, y no presentaron decoración. Éstos estuvieron asociados a la presencia de piedras, huesos, conchales y/o con alguna combinación de estos elementos, determinándose 6 tipos de nidos en función a la presencia y combinación de estos elementos. Aproximadamente el $92 \%$ de ellos estuvo asociado a la presencia de piedras. La distancia de los nidos respecto al mar varió de $1 \mathrm{~km}$ a $3.7 \mathrm{~km}$; mientras que la menor distancia entre dos nidos activos fue de 44.18 $\mathrm{m}$ y la mayor fue de $3.28 \mathrm{~km}$.

Considerando ambas temporadas, el $56.9 \%$ de los nidos fueron de 2 huevos, $41.4 \%$ de un huevo y $1.7 \%$ de 3 huevos. El éxito reproductivo fue de $55.6 \%$ ( $n=$ 27 nidos) para nidos en los que al menos eclosionó un huevo. Los pichones con una edad de máximo un día de eclosión presentaron un peso de $6.7 \mathrm{~g} \pm 0.54$ (rango: 6.0 a $7.3 \mathrm{~g}$ ), su cuerpo estaba cubierto de plumón con una ligera ausencia de éste en una parte del dorso. Las alas, cabeza y dorso presentaron plumón de color beige con puntos negros mientras que el pecho y vientre con plumón blanco.

El "gaviotín peruano" es un ave asincrónica en su reproducción mostrando fidelidad al nido y/o al lugar de anidación. Sin embargo, aun no se ha determinado si son los mismos individuos los que están reutilizando los nidos. El periodo reproductivo de esta especie se extendió desde noviembre hasta mayo, siendo más intenso durante los meses de verano. Las características del nido, huevos y pichones permiten que éstos se mimeticen en el desierto dificultando su hallazgo.

Se recomienda la realización de una investigación sobre las amenazas que afectan al "gaviotín peruano" en la colonia reproductiva de la península de Paracas con el objetivo de proponer las medidas respectivas que garanticen la conservación de esta especie amenazada.

\section{Agradecimientos.}

A María del Pilar Ramírez, Rachel Quispe, al personal de la RNP, Karin Obando, Liliana Ayala, Luis F. Felipe, Luis F. Cisneros, Luzdeli Cantoral, Sebastián Tapia, Ali Altamirano, Juan Guerra, Carlos A. Arnillas, Claudia Véliz, Carolina Tovar, Johann Pomar, Alessandro Catenazzi, Carlos Zavalaga y Carlos Guerra-Correa. A Martha Williams por su asesoría en la elaboración de la tesis: "Importancia de la Península de Paracas (Ica, Perú) en la Conservación del 'Gaviotín Peruano' Sternula lorata (Philippi y Landbeck, 1861)” y revisión del manuscrito.

El estudio fue financiado con fondos del Programa de Becas para Investigación de Especies Amenazadas "María Koepcke” de la Asociación Peruana para la Conservación de la Naturaleza - APECO y Conservación Internacional - CI (Amorós y Saravia, 2008).

\section{Literatura citada.}

Amorós S. \& Saravia P. 2008. Estado de Conservación de Sterna lorata, "Gaviotín Peruano" en la Reserva Nacional de Paracas (RNP), Ica - Perú”. Programa de Becas para la Investigación de Especies Amenazadas "Maria Koepcke” de APECO - CI. Lima - Perú. 
BirdLife International. 2009. Species factsheet: Sterna lorata. Consultado 3 ago. 2009. Disponible en http://www.birdlife.org.

Coker R. 1919. Habitats and economics relations of guano birds of Peru. Proceedings of Unites States National Museum. 56: 449 - 511.

Del Hoyo J., Elliott A. \& Sargatal J. 1996. Handbook of the birds of the World. Volumen 3: Hoatzin to Auks. Linx Editions. Barcelona.

Duffy D., Hays C. \& Plenge M. 1984. The conservation status of Peruvian seabirds. ICBP Technical Publication No. 2: $245-259$.

Decreto Supremo No 034-2004-AG El Peruano. Año XXI $\mathrm{N}^{\circ}$ 8859. Miércoles 22 de setiembre de 2004. Pág. 276853 - 276855. Consultado 1 de jul. 2009. Disponible en http://www.elperuano.com.pe/

Guerra-Correa C. 2003. Nidificación del Gaviotín Chirrío, Sterna lorata en Bahía Mejillones del Sur: Mitigación y manejo para la protección de las poblaciones locales. Corporación Nacional del Cobre de Chile, CODELCO FUREME. Informe 2002 Año 1. Antofagasta, Chile. 2005. Fauna de Vertebrados, Flora y Vegetación de la Desembocadura del Río Loa. I y II Región de Chile. Universidad de Antofagasta - Centro Regional de Estudios y Educación Ambiental (CREA). Chile.

Harrison P. 1985. Seabirds an identification guide. Houghton Mifflin Company Boston.

INRENA. 2002. Plan Maestro 2003 - 2007 de la Reserva Nacional de Paracas. Pisco - Perú.

Johnson A. 1967. The Birds of Chile and adjacent regions of Argentina, Bolivia y Peru. Volumen II. Buenos Aires.

Murphy R.C. 1936. Oceanic birds of South America. The Mc Millan Company, New York.
Reyes J. \& Hamman S. 2002. Paracas, nuestra Reserva: Información básica sobre la Reserva Nacional de Paracas. ACOREMA y WWF Oficina de Programa Perú.

Tello A., Quiñónez A. \& Merino P. 2008. Evaluación de la avifauna local y migratoria en los Humedales de Paraíso. Gerencia Regional Recursos Naturales y Gestión del Medio Ambiente. Huacho - Perú. Consultado 1 jul. $2009 . \quad$ Disponible en: http://www.regionlima.gob.pe/gerencias/rrnn/estudios/es tudio_avifauna_araiso.pdf

Vilina Y. 1998. Breeding observations of the Peruvian Tern in Chile. Colonial Waterbirds. 21 (1): 101-103.

Zavalaga C.B., Plenge M. \& Bertolero A. 2005. Estrategias de anidación y estado de conservación del Gaviotin Peruano Sterna lorata en el Perú. Pp. 83 en Libro de resúmenes VI Congreso Nacional de Ornitología Chiclayo, Perú. Riva-Melofiro, F. y A. More, eds. Chiclayo, Perú.

Plenge M. \& Bertolero A. 2008a. The breeding biology of the Peruvian Tern (Sternula lorata) in Perú. Waterbirds. 31 (4):550-560.

Plenge M. \& Bertolero A. 2008b. Nesting habitat and numbers of Peruavian Terns at five breeding sites in the central-southern coast of Peru. Ornitología Neotropical. 19:587-594.

Harvesty J., Mori G., ChávezVillavicencio C. \& Tello A. 2009. Current status of Peruvian Terns Sternula lorata in Peru: threats, conservation and research priorities. Bird Conservation International. 19:175-186.

\footnotetext{
${ }^{1}$ Afiliación cuando se realizó la investigación: Asociación Peruana para la Conservación de la Naturaleza APECO. Afiliación actual: APECO y Centro para la Conservación Integral de los Ecosistemas Marinos del Pacífico Este - ecOceánica. Dirección de correspondencia del autor principal: samorosk@yahoo.com

2 Reserva Nacional de Paracas. Servicio Nacional de Áreas Naturales Protegidas del Perú.

${ }^{3}$ Departamento de Biología, Facultad de Ciencias, Universidad Nacional Agraria La Molina.
} 